\title{
Extinction Conditions from a One-Sided Competition Model with a Holling Type I Functional Response for the Sterile Insect Technique
}

\author{
Yassine Chargui ${ }^{1,2}$, Anis Dhahbi ${ }^{1,2}$, Adel Trabelsi² \\ ${ }^{1}$ Physics Department, College of Science and Arts at Ar Rass, Qassim University, Ar Rass, Saudi Arabia \\ ${ }^{2}$ Unité de Recherche de Physique Nucléaire et des Hautes Energies, Faculté des Sciences de Tunis, Université de Tunis El Manar, \\ Tunis, Tunisie \\ Email: yassine.chargui@gmail.com, a.dhahbi@qu.edu.sa, adel.trabelsi@gmail.com
}

How to cite this paper: Chargui, Y., Dhahbi, A. and Trabelsi, A. (2018) Extinction Conditions from a One-Sided Competition Model with a Holling Type I Functional Response for the Sterile Insect Technique. Open Journal of Modelling and Simulation, 6, 13-26.

https://doi.org/10.4236/ojmsi.2018.62002

Received: February 4, 2018

Accepted: April 1, 2018

Published: April 4, 2018

Copyright (c) 2018 by authors and Scientific Research Publishing Inc. This work is licensed under the Creative Commons Attribution International License (CC BY 4.0).

http://creativecommons.org/licenses/by/4.0/

\begin{abstract}
The Sterile Insect Technique (SIT) is a powerful biological tool to control pest-populations. It could be integrated in management programmes of the Red Palm Weevil (RPW) Rhynchophorus ferrugineus Olivier. This pest threatens seriously date palms particularly in Saudi Arabia. As a matter of fact, the use of SIT has been very efficient in controlling RPW populations in coconut gardens in various countries. In this work, we outline the extinction conditions of a target wild-population using a one-sided competition model to describe its competition with released sterile insects. We employ a Holling type I functional response to describe the sterile-fertile one-sided competition and we consider two intra-specific competition sub-models, with and without Allee Effect, in modelling the growth of the wild-type population. We also study two manners of liberating sterile insects: single and periodic release strategies.
\end{abstract}

\section{Keywords}

Sterile Insect Technique, Mathematical Model, Extinction Conditions

\section{Introduction}

The Red Palm Weevil (RPW)Rhynchophorus ferrugineus Olivier is a notorious pest with a worldwide range and a history of fast spread and painful invasions to various countries, especially in the Arabian Peninsula [1] [2] [3]. It is still rated as one of the most destructive pest of palms in the world [4] [5], despite the best 
efforts of entomologists to develop effective control strategies against this weevil. In particular, approaches based on pheromone traps have been extensively employed to manage RPW [6] [7] [8] [9] [10]. Though they have lead to good results, they remain not fully efficient.

The interest to improve the efficiency of biological methods of insect pests control is being increased over the past few decades, trying to replace the classical insecticide-based ways. Hence, the sterile insect technique (SIT) has been proven to be an effective method in the field of the area-wide control of insects. The SIT method introduced by Knipling [11] [12] in 1930s, with a species-specific area-wide, is relying on the insect rearing, the sterilization without affecting the mating ability, and then the release of a specific numbers of insects [11] [13]. When they are released, the treated insects will compete for mates against the wild-type males, and when a wild-type female mates a released sterile male, it will have no progeny. Thus, the most of the wild-type females will mate with the released sterile males providing that sufficient mass of sterile insects is released in the field for a suitable enough period, so that no viable offspring will be produced. Finally, the control or indeed the eradication of the pests would be achieved in large areas due to the reduced fertility of the targeted insect.

Many studies have shown that the SIT method could be successfully operated against the most important agricultural pests around the world, such as Cochliomyia hominovorrax, the Mediterranean fruit fly (Ceratitis capitata) [14] [15], the New World screwworm, and the tsetse fly Glossina spp. In addition, an efficiently control of the RPW in the Indian coconut gardens has been proven in papers [16] [17] by adopting the SIT strategy. Therefore, it becomes widely believed that the SIT has to be considered as a major part of the pest insects management programs [18].

The purpose of this paper is to introduce an impartially analytical model for the SIT leading to precise mathematical conditions for the eradication process through a theoretical treatment of suppression strategies. In particular, the minimal size of sterile males to be released in order to achieve the extinction of the pest population will be determined for different strategies. This aids in estimating the cost of applying the SIT program. Several categories of models have been applied the Sterile Insect Technique in the literature, such as continuous-time, deterministic and discrete-time models where the corresponding advantages are explained in Ref. [19]. In addition to their generality, the analytic models lend a high precision to the prediction of the different parameters. However, the simplification of such analytic models, needed to be mathematically tractable, would represents their major weakness.

The paper is organized as follows. In Section 2, we present our mathematical model for the SIT. In Sections 3, the model is used to study the conditions to achieve total extinction of the target population for two release strategies of sterile males. We consider two release strategies; single and periodic and we treat two sub-models for intra-specific competition, with an without Allee effect. In 
the final section we give our conclusion.

\section{The Mathematical Model}

In this work we are going to consider a deterministic single-specie model assuming continuous-time and density-dependent growth of the wild-type population [20]. Spatial movement and effects of environmental factors and others are not included. The model also assumes that released sterile insects are male-only. Since the SIT emphasis juste the competition for mates, which affects only the fertile population, and admitting that the presence of fertile insects does not affect the sterile population, neither through the competition for mates nor through the competition for natural resources (no larval survivorship [21]), we conclude that a one-sided competition which affects solely wild-type insects is convenient for SIT modelling. Explicitly, the model we suggest is described by the following nonlinear dynamic system:

$$
\begin{gathered}
\frac{\mathrm{d} M}{\mathrm{~d} t}=-\frac{M}{\tau_{s}}+R(t) \\
\frac{\mathrm{d} N}{\mathrm{~d} t}=r N F(N)-\lambda M(t) H(N(t))
\end{gathered}
$$

where $N(t)$ and $M(t)$ are respectively, wild-type and sterile populations sizes. $R$ is a function characterizing the release strategy of sterile males which have an average life-time $\tau_{s}$. The term $r F(N)$ is the per capita growth rate of wild-type population. The one-sided competition between sterile and fertile insects is described by the term $\lambda M(t) H(N(t))$ where $H(N)$ is the functional response. The parameter $\lambda$ is a proportionality constant set by the rate of interaction between sterile males and fertile females. As we shall see later, the mathematical tractability and simplicity of this simple one stage model, with no other species interactions, justifies its choice. We shall consider two forms for the intra-specific competition model. The first form of $F$ corresponds to the logistic growth law and reads:

$$
F_{1}(N)=1-N / K
$$

where $K$ represents the carrying capacity (the maximum number of individuals that the environment can stably support) in the absence of sterile males. Notice that $F_{1}$ is normalized, i.e., $\max _{N} F_{1}=1$. The second form of $F$ aims to take into account the impact of the presence of an Allee effect [22] [23] [24], and is given by:

$$
F_{2}(N)=k_{2}(\alpha-N)(1-N / K)
$$

The Allee effect emphasis the possibility of positive relationship between intra-competition and the population size [25] [26]. This is an important dynamic phenomenon especially for low-density populations [27] [28] [29] [30] [31], and may particularly increase their extinction risk [28] [29] [32] [33]. This effect may have different causes. The most obvious is the difficulty to find mates 
at low population sizes [34]. Less important is the necessity of a minimal group size to rear offspring, seek food and sustain predator attacks. Notice that the parameter $\alpha$ in Equation (4) represents the "Allee effect constant". As a matter of fact the Allee effect will be more stronger as $\alpha$ is bigger. In addition, we assume a strong Allee effect, which occurs when the condition $0<\alpha<\beta$ is fulfilled [22]. $k_{2}$ is a constant set at $k_{2}=4 K /(\alpha-K)^{2}$ to meet the normalization condition $\max _{N} F_{2}=1$. Behaviors of intra-specific sub-models (3) and (4) are shown in Figure 1.

\section{Interspecific Competition}

As mentioned above, the functional $H(N)$ appearing in Equation (2), describes the response of the fertile population to the presence imposed of sterile insects. In this work we shall assume this response to be of Holling Type I, i.e., $H(N)=N$ [35] [36]. That is a linear relationship between sterile-fertile mating-rate and the size of fertile population.

In the next section, we shall see that Equation (2) is exactly solvable for the logistic sub-models (3) in both cases of single and periodic release strategies. This solution allows to access the exact extinction condition. However this is not the case when the presence of an Allee effect is taken into account. In this situation,the condition for total extinction shall be obtained from an analytical approximate solution of the model derived using a perturbation approach. For both cases with and without Allee effect, we suppose that the wild-type population is in a its stable steady state, before the release of sterile males, i.e., $N(0)=K$.

\section{Study of Extinction Conditions}

\subsection{Logistic Sub-Model}

In case when a logistic growth law of the wild-type population is assumed,

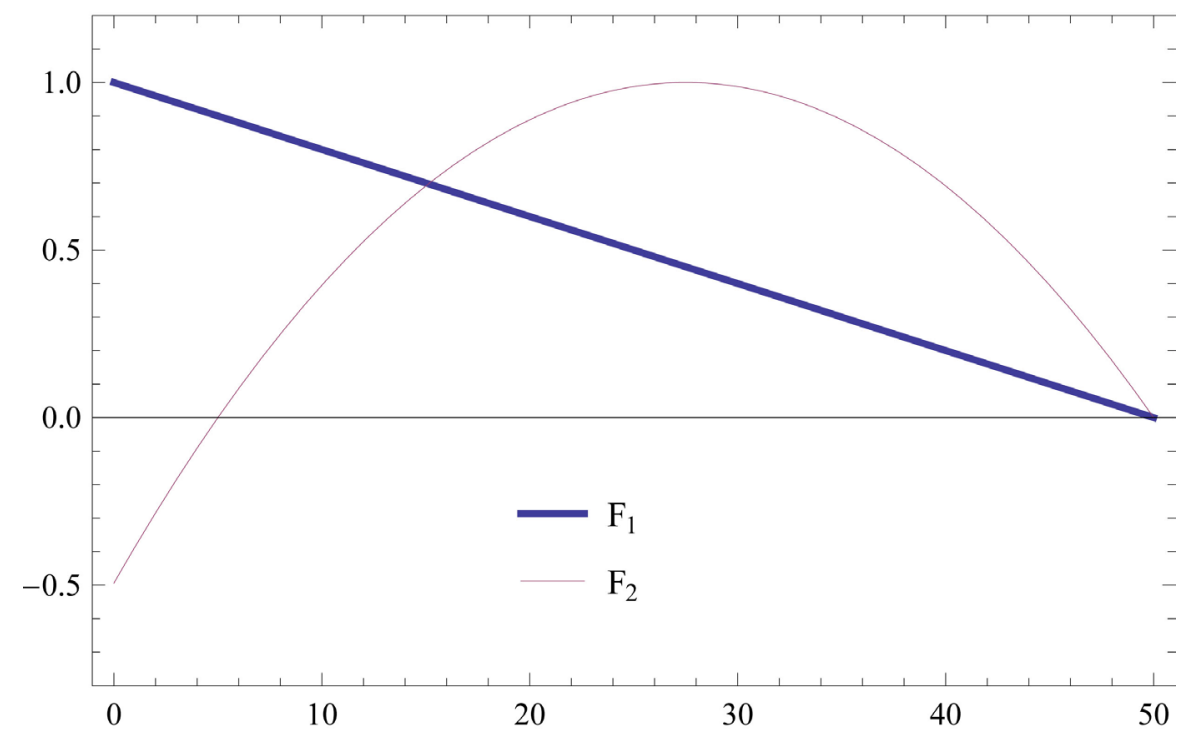

Figure 1. Plots of intra-specific growth sub-models $F_{1}$ and $F_{2}$ for $K=50$ and $\alpha=5$. 
Equation (2) takes the form:

$$
\frac{\mathrm{d} N}{\mathrm{~d} t}=r N(1-N / K)-\lambda M(t) N
$$

An exact analytical solution for this equation can be straightforwardly constructed as follows. Making the change $N=1 / z$, we get the following linear first order equation for $z(t)$ :

$$
\frac{\mathrm{d} z}{\mathrm{~d} t}+(r-\lambda M(t)) z=r / K
$$

The complete solution for this equation with the initial condition $N(0)=K$ is given as:

$$
z(t)=\left\{\frac{1}{K}+\frac{r}{K} \int_{0}^{t} \exp \left[r t^{\prime}-\lambda \int_{0}^{t^{\prime}} M(x) \mathrm{d} x\right] \mathrm{d} t^{\prime}\right\} \times \exp \left[-r t+\lambda \int_{0}^{t} M(x) \mathrm{d} x\right](7)
$$

The success or not of an SIT control strategy depends on various factors with varying significance, particularly on the choice of the mass release strategy of sterile males. Therefore we shall investigate conditions of success of the SIT control method for both single and periodic release strategies. This allows to shed light on the relevance of the release strategy for parameters determining the effectiveness the SIT method.

\subsubsection{Single Release Strategy}

In fact, single release strategy is not usually practiced, however its study could help in fixing relevant parameters of the SIT method that should be taken into consideration when studying the periodic release case. In the case of single release, the sterile population size obeys the law:

$$
\frac{\mathrm{d} M}{\mathrm{~d} t}=-\frac{M}{\tau_{\mathrm{s}}}
$$

This means that the number of sterile insects decays exponentially with a characteristic time equal to their average lifetime $\tau_{s}$ :

$$
M(t)=M_{0} \mathrm{e}^{-\frac{t}{\tau_{s}}}
$$

where $M_{0}$ is the initial released number of sterile males. Next we shall investigate conditions for that SIT reduces the wild-type population size to zero within a time scale $\tau_{e}$. In addition, since only the released generation of sterile males intervenes in this strategy, $\tau_{e}$ should be of the same order of $\tau_{s}$. Now we have

$$
N(t)=\left\{\frac{1}{K}+\frac{r}{K} \int_{0}^{t} \exp \left[r t^{\prime}-\frac{\lambda M_{0}}{\tau_{s}}\left(1-\mathrm{e}^{-\frac{t^{\prime}}{\tau_{s}}}\right)\right] \mathrm{d} t^{\prime}\right\}^{-1} \times \exp \left[r t-\frac{\lambda M_{0}}{\tau_{s}}\left(1-\mathrm{e}^{-\frac{t}{\tau_{s}}}\right)\right]
$$

The asymptotic behaviour of the previous function as $t \rightarrow \infty$ shows that the extinction cannot achieved with the single release strategy for the Logistic Sub-model $F_{1}$. As a matter of fact, it is straightforward to verify using elementary mathematical tools that 


$$
N(t) \geq \frac{\exp \left[r t-\frac{\lambda M_{0}}{\tau_{s}}\left(1-\mathrm{e}^{-\frac{t}{\tau_{s}}}\right)\right]}{\frac{1}{K}+\frac{r}{K} \int_{0}^{t} \mathrm{e}^{r t^{\prime}} \mathrm{d} t^{\prime}}
$$

Then, by taking the limit $t \rightarrow \infty$ we find that $N_{\min }=K \mathrm{e}^{-\frac{\lambda M_{0}}{\tau_{s}}}$ is a lower bound for the size of the wild population as $t \rightarrow \infty$. This proves the non-extinction of the pest population. This behaviour of the size of the wild-type population may be explained qualitatively as follows. An effective extinction requires a persistent negative growth rate of the wild population: $\frac{\mathrm{d} N}{\mathrm{~d} t}<0$. However this could not be satisfied for sufficiently large time $t$. As the number of sterile insects decreases exponentially, it will certainly goes down under the a level permitting to the wild population to recover a persistent positive growth rate and attend again a stable carrying-capacity state. Later we shall see that the presence of a strong Allee effect allows to reach effective extinction with the Logistic Sub-model and a single release strategy. In Figure 2 we display the behavior of the wild type population $N(t)$ for the sub-model $F_{1}$, in the case of single release strategy.

\subsubsection{Periodic Release Strategy}

The periodic release strategy can better models realistic situations. In this case, the dynamic of the sterile population size is dictated by the equation:

$$
\frac{\mathrm{d} M}{\mathrm{~d} t}=-\frac{M}{\tau_{s}}+R_{0}(1+\epsilon \cos (\omega t+\phi))
$$

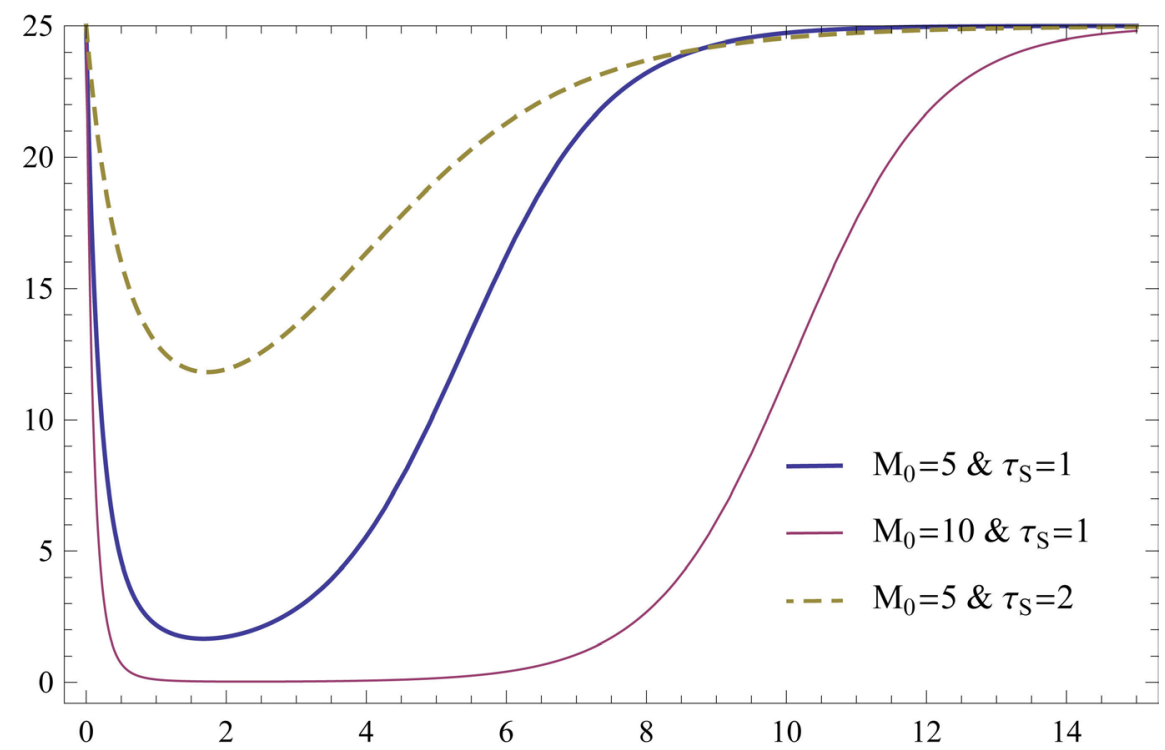

Figure 2. Illustrations of the behaviour of $N(t)$ for the sub-model $F_{1}$ in the cases of single release strategy. Solid (broken) lines correspond to $M_{0}=20 \quad\left(M_{0}=5\right)$. Other parameters values are $K=25$ and $\lambda=1$. 
where $\epsilon, \omega$ and $\phi$ are independent parameters with $0<\epsilon \leq 1$. Here we can disregard the transitory solution which decays exponentially. Then we are left with

$$
M(t)=\tau_{s} R_{0}\left[1+\frac{\epsilon}{1+\tau_{s}^{2} \omega^{2}} \cos (\omega t+\phi)+\frac{\epsilon \tau_{s} \omega}{1+\tau_{s}^{2} \omega^{2}} \sin (\omega t+\phi)\right]
$$

The last form of $M(T)$ results in the following expression for the wild-type population

$N(t)=\left\{\frac{1}{K}+\frac{r}{K} \int_{0}^{t} \exp \left[r t^{\prime}+\lambda\left(h(0)-h\left(t^{\prime}\right)\right)\right] \mathrm{d} t^{\prime}\right\}^{-1} \times \exp [r t+\lambda(h(0)-h(t))]$

with

$$
h(t)=\tau_{s} R_{0}\left[t+\frac{\epsilon / \omega}{1+\tau_{s}^{2} \omega^{2}} \sin (\omega t+\phi)-\frac{\epsilon \tau_{s}}{1+\tau_{s}^{2} \omega^{2}} \cos (\omega t+\phi)\right]
$$

In comparison with the first case, $\epsilon, \omega$ and $\phi$ are additional parameters that should be considered for the periodic release strategy. In particular, the extinction dynamics depends on the relative time scales described by $\omega \tau_{s}$ and $\omega \tau_{e}$. In this strategy, its well understood that the extinction time $\tau_{e}$ will clearly exceed the time-time $\tau_{s}$. Furthermore, the strategy is usually practiced with a period close to the life-time $\tau_{s}$, thus the number $\omega \tau_{s}$ will have the order of some units. To emphasize the change in the behaviour of the pest-population size with the periodic release compared to the case of single release, we plot in Figure 3 the function $N(t)$ given by Equation (14) for some illustrative parameters of the method.

Next, expression (14) will be the starting point for our investigation of

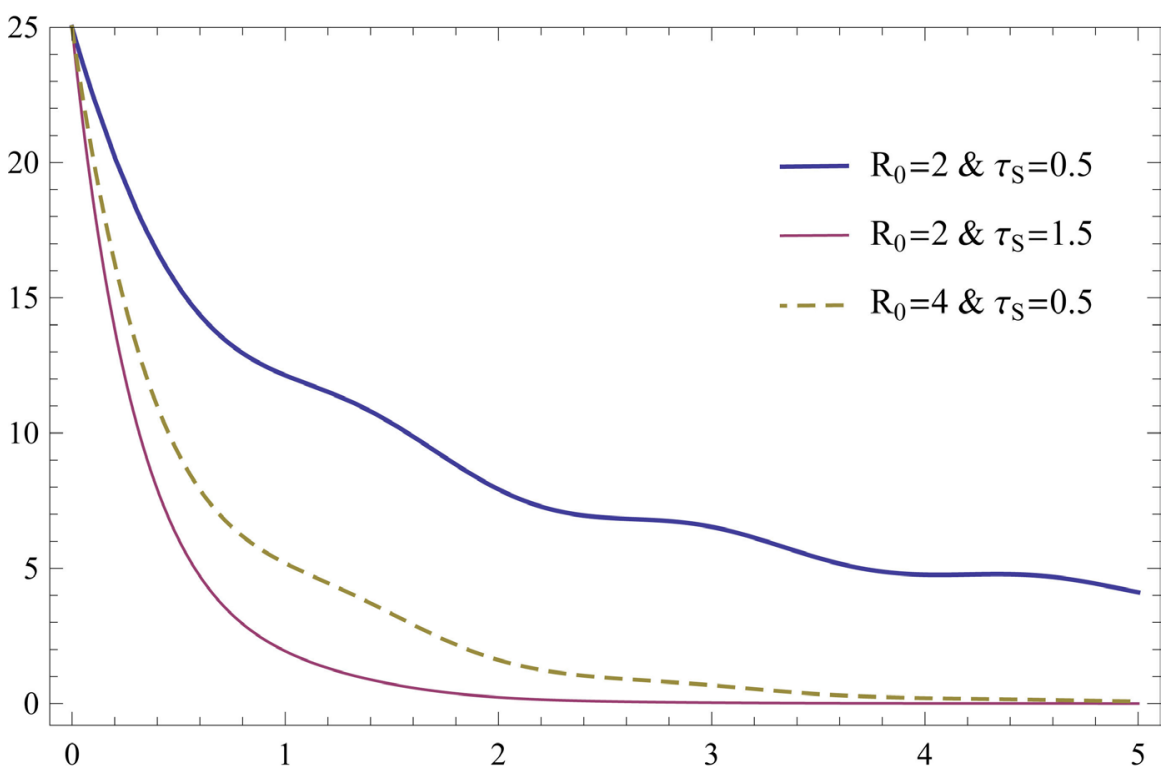

Figure 3. Illustrations of the behaviour of $N(t)$ for the sub-model $F_{1}$ in the cases of periodic release strategy for some illustrative crucial parameters. Others parameters values are $K=25$ and $\lambda=1, \omega=4, \epsilon=0.5$ and $\phi=0$. 
extinction conditions. In particular it will be used to obtain the threshold density of sterile males, $\left(R_{0 T}\right)$, to be released to ensure the eradication of the wild population in a given time $\tau_{e}$. This threshold size is believed to be crucial for any SIT program. Results obtained from expression (14) for the extinction condition and its variations with the relevant parameters of the method are summarized in Figure 4.

\subsection{Including Allee Effect}

As said before, the presence of an Allee effect may substantially modify the dynamic of the pest population especially at low sizes. Particularly, if such effect is present, we merely have to decrease the wild population size from $K$ to $\alpha$ in order to achieve total extinction. For the intra-specific sub-model $F_{2}$, Equation (2) takes the form:

$$
\frac{\mathrm{d} N}{\mathrm{~d} t}=\bar{r} N(N-\alpha)(1-N / K)-\lambda M(t) N
$$

with $\bar{r}=r k_{2}$. To the best of our knowledge, an exact analytical solution for this equation cannot be obtained, at least for the two froms of the function $M(t)$ we are considering. Therefore we shall use a perturbative method to handle this equation. Making the change of function: $N=1 / z$, we obtain for $z(t)$ the following equation:

$$
\frac{\mathrm{d} z}{\mathrm{~d} t}=\frac{\bar{r}}{K} \frac{1}{z}-\bar{r}\left(\frac{\alpha}{K}+1\right)+(\lambda M(t)+\alpha \bar{r}) z
$$

As $K$ represents the initial equilibrium size of the pest population, it is legitimate to assume that $1 / K \ll 1$. If we additionally assume that $(K-\alpha)$ is

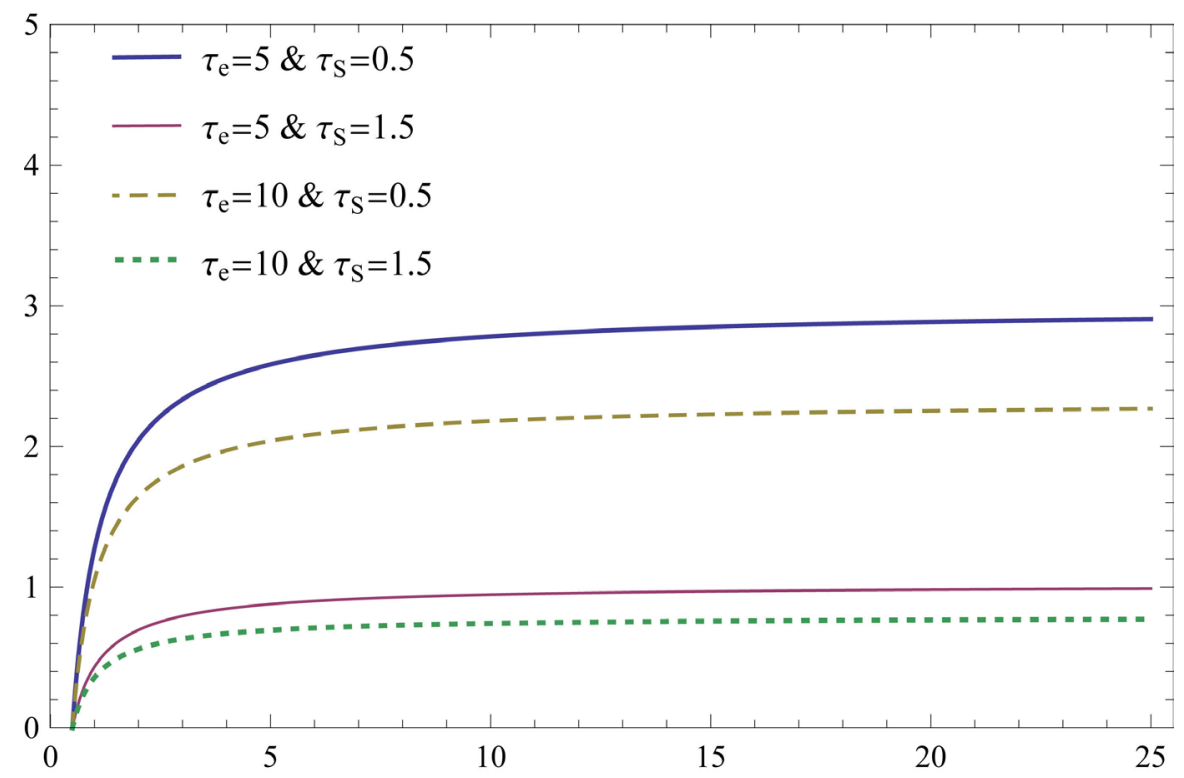

Figure 4. The critical density of sterile males $\left(R_{0 T}\right)$, as a function of the wild-population size for some illustrative crucial parameters. Others parameters values are $\lambda=1, \omega=4$, $\epsilon=0.5$ and $\phi=0$. 
of the same order as $K$ (the condition which applies to realistic situation), we can infer that the term proportional to $1 / z$ is negligible compared with other terms in the r.h.s of Equation (20). Then we can conclude that the function $z_{0}(t)$, solution of the equation

$$
\frac{\mathrm{d} z_{0}}{\mathrm{~d} t}-(\alpha \bar{r}+\lambda M(t)) z_{0}=-\bar{r}\left(\frac{\alpha}{K}+1\right)
$$

constitutes a good first approximation to the function $z(t)$. We can remark that the dynamics of $z_{0}(t)$ follows an equation having the same form as Equation (6) which is exactly solvable. The general form of $z_{0}(t)$ will be similar to Equation (7) and it will be given bellow. Moreover, as $z(t)$ is close to $z_{0}(t)$, we have $\left|z_{0}-z / z_{0}\right| \ll 1$. Then it is possible to improve our approximation for the solution $z(t)$ by expanding the term in $1 / z$ in Equation (20) as:

$$
\frac{1}{Z}=\frac{1}{z_{0}}\left(1+\frac{Z-z_{0}}{z_{0}}\right)^{-1} \approx \frac{1}{z_{0}}\left(2-\frac{Z}{z_{0}}\right)+\cdots
$$

Then, the resulting equation for $z(t)$ reads

$$
\frac{\mathrm{d} z}{\mathrm{~d} t}=\bar{r}\left(\frac{2}{K z_{0}}-\frac{\alpha}{K}-1\right)+\left(\lambda M(t)+\alpha \bar{r}-\frac{\bar{r}}{K z_{0}^{2}}\right) z
$$

In order to emphasis the relation between $z_{0}(t)$ and $z(t)$, we will seek a general solution for the previous equation having the form $z(t)=z_{0}(t)+y(t)$, with $y(t)$ an unknown function. Substituting this form in Equation (20), we get to the following equation for $y(t)$

$$
\frac{\mathrm{d} y}{\mathrm{~d} t}=\frac{\bar{r}}{K z_{0}}+\left(\lambda M(t)+\alpha \bar{r}-\frac{\bar{r}}{K z_{0}^{2}}\right) y
$$

where we have made use of Equation (20) satisfied by $z_{0}(t)$. The last equation leads to the following form for the function $y(t)$

$$
\begin{aligned}
y(t)= & \frac{\bar{r}}{K}\left\{\int_{0}^{t} \frac{1}{z_{0}\left(t^{\prime}\right)} \exp \left[-\alpha \bar{r} t^{\prime}-\int_{0}^{t^{\prime}}\left(\lambda M(x)-\frac{\bar{r}}{K z_{0}^{2}(x)}\right) \mathrm{d} x\right] \mathrm{d} t^{\prime}\right\} \\
& \times \exp \left[\alpha \bar{r} t+\int_{0}^{t}\left(\lambda M(x)-\frac{\bar{r}}{K z_{0}^{2}(x)}\right) \mathrm{d} x\right]
\end{aligned}
$$

where the expression of $z_{0}(t)$ is given by

$$
\begin{aligned}
z_{0}(t)= & \left\{\frac{1}{K}-\bar{r}\left(\frac{\alpha}{K}+1\right) \int_{0}^{t} \exp \left[-\alpha \bar{r} t^{\prime}-\lambda \int_{0}^{t^{\prime}} M(x) \mathrm{d} x\right] \mathrm{d} t^{\prime}\right\} \\
& \times \exp \left[\alpha \bar{r} t+\lambda \int_{0}^{t} M(x) \mathrm{d} x\right]
\end{aligned}
$$

The integration constant has been fixed using the condition $z(0)=1 / K$. As we are considering only realistic cases where $z_{0}(t)>0$, we can see from Equation (22) that $y(t)$ is always positive and this means that $z(t)>z_{0}(t)$ which means that the sizes $N_{0}(t)$ and $N(t)$ resulting respectively, from the 
solutions $z_{0}(t)$ and $z(t)$ are such that $N(t)<N_{0}(t)$. A comparison between $N_{0}(t)$ and $N(t)$ for the single release strategy, is shown in Figure 5. We can clearly see that $N(t)$ presents a faster decay than the function $N_{0}(t)$, and this is related to the manifestation of Allee effect which is more important in the case of the size $N(t)$.

Let us note that the high complication of the last expression of $z(t)$ (compared with expression (7)) renders its analytical handling very difficult. We shall use Mathematica toolkit to extract all the information from it. As we shall see later, the presence of Alle effect drastically affects the large-time behaviour of the pest population size, especially in the case of single release strategy. Next we shall investigate the implications of expressions (22) and (23) for extinction conditions in the two cases of release strategies. Notice that in the case of single release strategy, $M(t)$ should be substituted by its form (9), while for a periodic release strategy, $M(t)$ should be replaced by its expression (13). Results implied by expressions (22) and (23) for the extinction condition and its variations with the relevant parameters of the method are summarized in Figure 6 \& Figure 7.

\section{Conclusions}

To sum up, the paper studies analytically the outcomes of a non-spatial deterministic model for the Sterile Insect Technique. Two sub-models for the intra-specific competition have been considered. The first assumes a logistic growth rate while the second takes into account the presence of an eventual Allee effect. Moreover, the response functional, describing the interspecific competition have been assumed to be Holling Type I. This allows a substantial

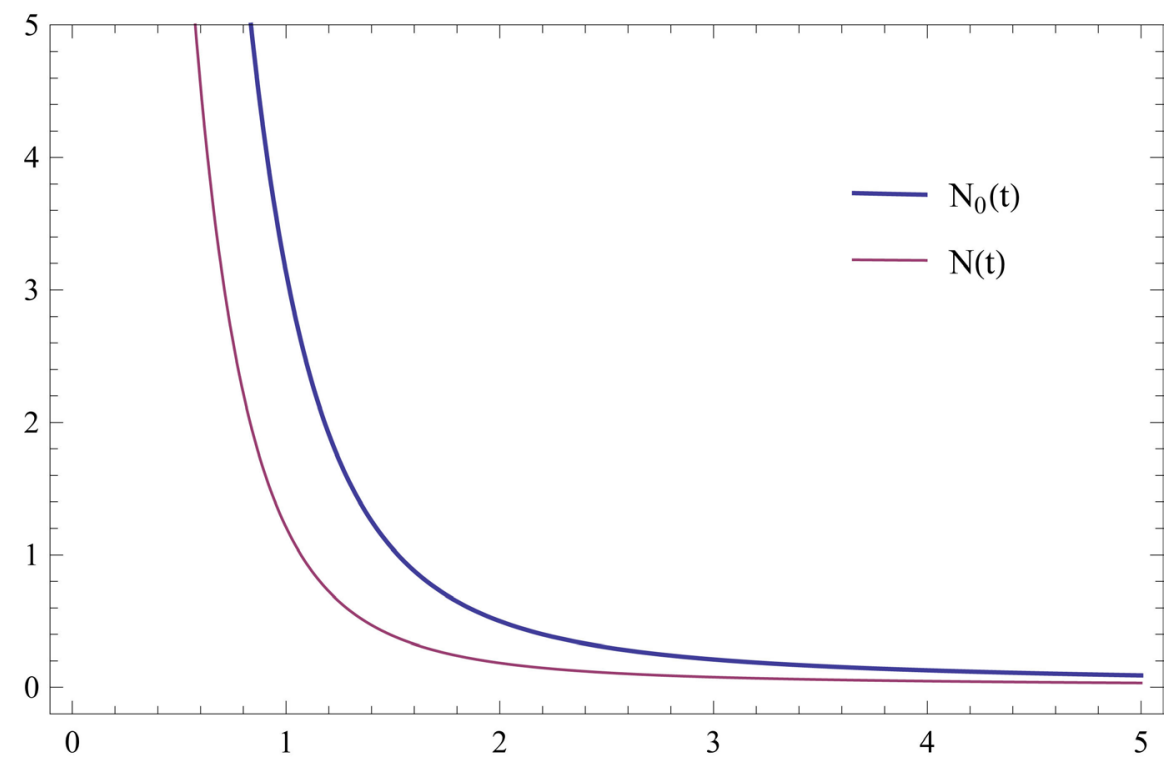

Figure 5. Illustrative decays of wild populations sizes $N(t)$ and $N_{0}(t)$ for the single release strategy. Others parameters values are $\lambda=1, \epsilon=1, \alpha=5, M_{0}=7$ and $k=80$. 


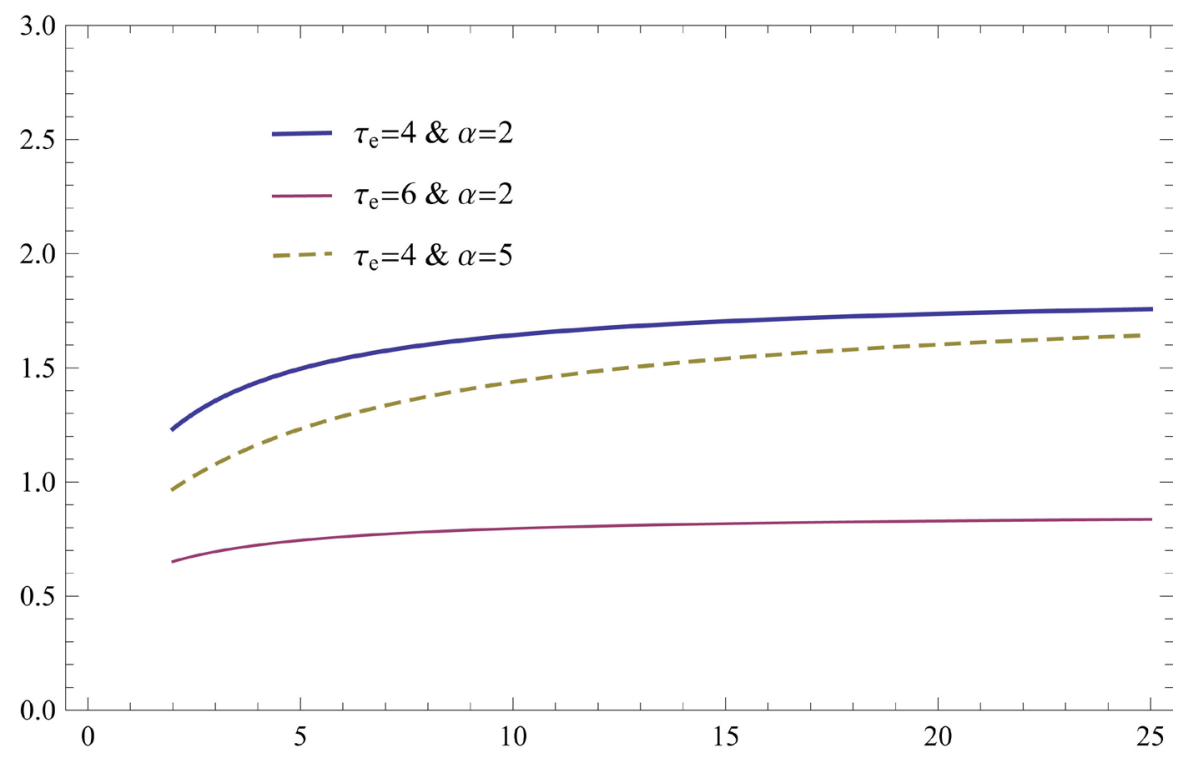

Figure 6. The critical density of sterile males $\left(R_{0 T}\right)$, as a function of the wild-population size, for single release strategy, is sketched for some illustrative crucial parameters. Others parameters values are $\lambda=1$ and $r=1$.

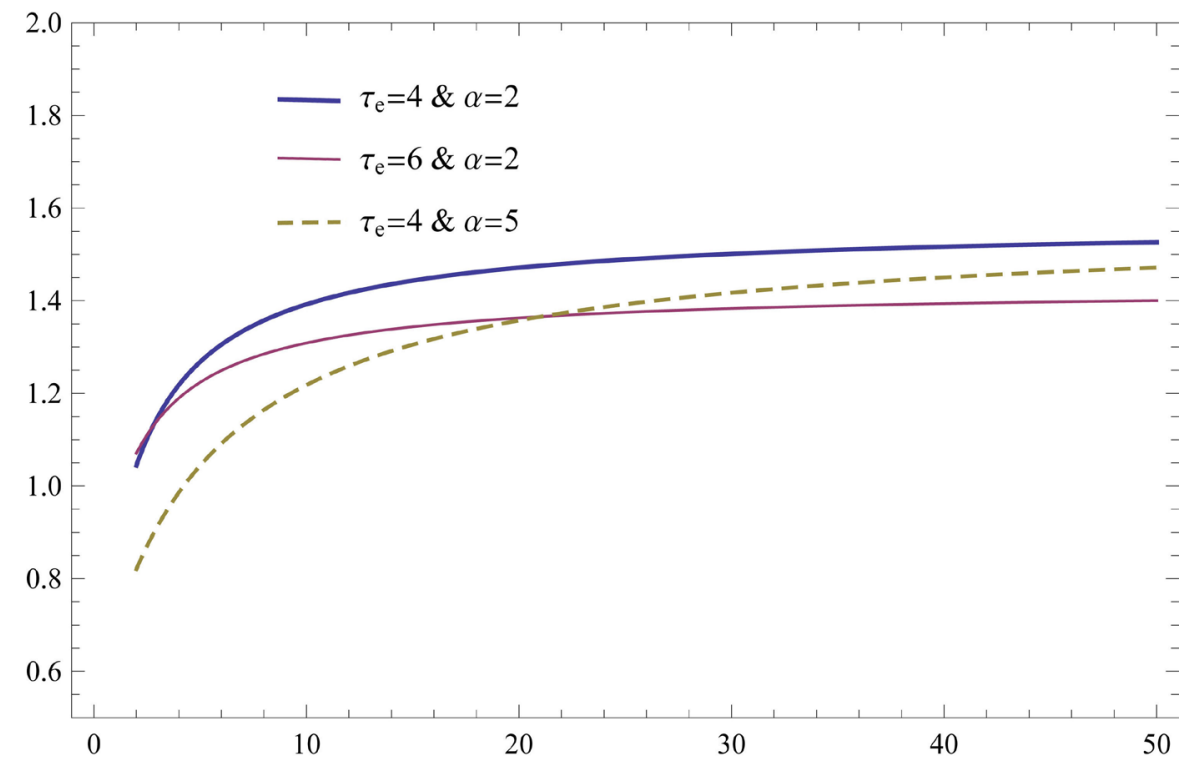

Figure 7. The critical density of sterile males $\left(R_{0 T}\right)$, as a function of the wild-population size, for periodic release strategy, is sketched for some illustrative crucial parameters. Others parameters values are $\lambda=1, r=1, \omega=2, \phi=0$ and $\epsilon=0.5$.

simplification of the mathematical study of the SIT dynamics. As a matter of fact equation describing this dynamics has been handled analytically. Despite its simplicity the proposed model reproduces satisfactory results. This model permits also a direct implementation of the mass-release strategy. In our study two manner of liberating sterile males have been considered; the single release strategy and the more realistic periodic release strategy.

Results of the study indicate that eradication of the pest population requires a 
minimal (critical) size of sterile released males. We have determined this critical size for various for the two considered growth sub-models when the release strategy, single or periodic, allows the achievement of extinction. In addition, the study has allowed to highlight the most important parameters intervening in deciding the success or not of a SIT program. Particularly, the model yields an impossibility of achieving eradication of the wild population experiencing a logistic growth rate when the sterile-fertile interaction is described with a Holling Type I response.

Despite of the above mentioned satisfactory findings yielded by the presented model, the latter is far a way from being complete and remains a first approximation for the SIT dynamics which is actually more complicated. Indeed, real practiced SIT programs may face several factors of difficulty such as lack of competitiveness of released weevils, environmental conditions, migration of insects, bad timing of release, etc, that may be crucial for the success of the program. However, we should admit that the inclusion of all these factors in modeling SIT is not possible. After all, we think that despite the simplicity of mathematical model it uses, this study allows to fix elements to be considered before deciding strategies to adopt for any SIT programme, which may be crucial for its success. Of course, a good decision made signifies particularly a reduce of the cost of the SIT program. This may be constructive for the manage of RPW populations in farms and urban palms in Saudi Arabia.

\section{Acknowledgements}

The authors gratefully acknowledge Qassim University, represented by the Deanship of Scientific Research, on the material support for this research under the number (3390) during the academic year $1436 \mathrm{AH} / 2015 \mathrm{AD}$.

\section{References}

[1] Morici, C. (1998) Phoenix Canariensis in the Wild. Principles, 42, 85-93.

[2] Abraham, V.A., Al-Shuaibi, M.A., Faleiro, J.R., Abozuhairah, R.A. and Vidyasagar, P.S.P.V. (1998) An Integrated Management Approach for Red Palm Weevil Rhynchophorus ferrugineus Oliv: A Key Pest of Date Palm in the Middle East. Agriculture Science, 3, 77-83.

[3] Fiaboe, K.K.M., Peterson, A.T., Kairo, M.T.K. and Roda, A.L. (2012) Predicting the Potential Worldwide Distribution of the Red Palm Weevil Rhynchophorus ferrugineus (Olivier) (Coleoptera: Curculionidae) Using Ecological Niche Modeling. Florida Entomologist, 95, 659-673. https://doi.org/10.1653/024.095.0317

[4] EPPO (European and Mediterranean Plant Protection Organization) (2008) Data Sheets on Quarantine Pests Rhynchophorus ferrugineus. EPPO Bulletin, 38, 55-59.

[5] EPPO (2009) Rhynchophorus ferrugineus Found on Howea forsteriana in Sicilia, Italy. No. 3 2009/051. European and Mediterranean Plant Protection Organization, Paris.

[6] Faleiro, J.R. and Chellappan, M. (1999) Attraction of Red Palm Weevil Rhynchophorus ferrugineus to Different Ferrugineol Based Pheromone Lures in Coconut Gardens. Journal of Tropical Agriculture, 37, 60-63. 
[7] Faleiro, J.R. (2006) A Review of the Issues and Management of the Red Palm Weevil Rhynchophorus ferrugineus (Coleoptera: Rhynchophoridae) in Coconut and Date Palm during the Last One Hundred Years. International Journal of Tropical Insect Science, 26, 135-154.

[8] Al-Shawaf, A.M., Al-Shagag, A., Al-Bagshi, M., Al-Saroj, S., Al-Bather, S., Al-Dandan, A.M., Abdallah, A.B. and Faleiro, J.R. (2013). A Quarantine Protocol against Red Palm Weevil Rhynchophorus ferrugineus (Olivier) (Coleoptera: Curculionidae) in Date Palm. Journal of Plant Protection Research, 53, 409-415. https://doi.org/10.2478/jppr-2013-0061

[9] Faleiro, J.R. (2005) Pheromone Technology for the Management of Red Palm Weevil Rhynchophorus ferrugineus (Olivier) (Coleoptera: Rhynchophoridae). A Key Pest of Coconut. Technical Bulletin, 4, 40 p.

[10] Huang, S.C., Ma, Z.L., Qin, W.Q., Li, C.X., Yu, F.Y. and Han, C.W. (2008) The Trapping Effect of Aggregation Pheromone for the Red Palm Weevil (Rhynchorus ferrugineus (Olivier)) and Its Traps Development. China Forestry Science and Technology.

[11] Knipling, E.F. (1955) Possibilities of Insect Control or Eradication through the Use of Sexually Sterile Males. Journal of Economic Entomology, 48, 459-462. https://doi.org/10.1093/jee/48.4.459

[12] Knipling, E.F. (1959) Screwworm Eradication: Concepts and Research Leading to the Sterile Male Method. Smithsonian Institution, Washington DC, 409-418.

[13] Krafsur, E.S. (1998) Sterile Insect Technique for Suppressing and Eradicating Insect Population: 55 Years and Counting. Journal of Agricultural Entomology, 15, 303-317.

[14] Robinson, A.S. (2002) Mutations and Their Use in Insect Control. Mutation Research/Reviews in Mutation Research, 511, 113-132. https://doi.org/10.1016/S1383-5742(02)00006-6

[15] Wong, T.T.Y., Kobayashi, R.M. and McInnis, D.O. (1986) Mediterranean Fruit Fly (Diptera: Tephritidae): Methods for Assessing the Effectiveness of Sterile Insect Technique Releases. Journal of Economic Entomology, 79, 1501-1506. https://doi.org/10.1093/jee/79.6.1501

[16] Rahalkar, G.W., Harwalkar, M.R., Rananvare, H.D., Shantaram, K. and Goplayengar, A.R. (1973) Laboratory Studies on Radiation Sterilization of the Red Palm Weevil, (Rhynchophorus ferrugineus Oliv.) Males. Journal of Plantation Crops, 1 , 141-145.

[17] Rahalkar, G.W., Harwalkar, M.R., Rananvare, H.D., Kurian, C., Abrham, V.A. and Koya, A.K.M. (1977) Preliminary Field Studies on the Control of the Red Palm Weevil, Rhynchophorus ferrugineus Using Radio Sterilized Males. Journal of Nuclear Agriculture and Biology, 6, 65-68.

[18] Krishnakumar, R. and Maheswari, P. (2007) Assessment of the Sterile Insect Technique to Manage Red Palm Weevil Rhynchophorus ferrugineus in Coconut. In: Vreysen, M.J.B., Robinson, A.S. and Hendrichs, J., Eds., Area-Wide Control of Insect Pests, Springer, Dordrecht, 475-485.

https://doi.org/10.1007/978-1-4020-6059-5_44

[19] Costello, W.G. and Taylor, H.M. (1975) Mathematical Models of the Sterile Male Technique of Insect Control. In: Charnes, A. and Lynn, W.R., Eds., Mathematical Analysis of Decision Problems in Ecology, Springer, Berlin, Heidelberg, 318-359. https://doi.org/10.1007/978-3-642-80924-8_12

[20] Barclay, H. and Mackauer, M. (1980) The Sterile Insect Release Method for Pest 
Control: A Density-Dependent Model. Environmental Entomology, 9, 810-817. https://doi.org/10.1093/ee/9.6.810

[21] Miller, D.R. and Weidhaas, D.E. (1974) Equilibrium Populations during a Sterile-Male Release Program. Environmental Entomology, 3, 211-216.

[22] Lewis, M.A. and Kareiva, P. (1993) Allee Dynamics and the Spread of Invading Organisms. Theoretical Population Biology, 43, 141-158. https://doi.org/10.1006/tpbi.1993.1007

[23] Owen, M.R. and Lewis, M.A. (2001) How Predation Can Slow, Stop or Reverse a Prey Invasion. Bulletin of Mathematical Biology, 63, 655-684. https://doi.org/10.1006/bulm.2001.0239

[24] Wang, M.E. and Kot, M. (2001) Speeds of Invasion in a Model with Strong or Weak Allee Effects. Mathematical Biosciences, 171, 83-97. https://doi.org/10.1016/S0025-5564(01)00048-7

[25] Allee, W.C. (1931) Animal Aggregations: A Study in General Sociology. University of Chicago Press, Chicago, IL. https://doi.org/10.5962/bhl.title.7313

[26] Allee, W.C. (1938) The Social Life of Animals. Heinemann, London. https://doi.org/10.5962/bhl.title.7226

[27] Asmussen, M.A. (1979) Density-Dependent Selection II. the Allee Effect. The American Naturalist, 114, 796-809. https://doi.org/10.1086/283529

[28] Lande, R. (1987) Extinction Thresholds in Demographic Models of Territorial Populations. The American Naturalist, 130, 624-635. https://doi.org/10.1086/284734

[29] Dennis, B. (1989) Allee Effects: Population Growth, Critical Density, and the Chance of Extinction. Natural Resource Modeling, 3, 481-538.

https://doi.org/10.1111/j.1939-7445.1989.tb00119.x

[30] Lande, R., Engen, S. and Saether, B.E. (1994) Optimal Harvesting, Economic Discounting and Extinction Risk in Fluctuating Populations. Nature, 372, 88-90. https://doi.org/10.1038/372088a0

[31] Stephan, T. and Wissel, C. (1994) Stochastic Extinction Models Discrete in Time. Ecological Modelling, 75-76, 183-192. https://doi.org/10.1016/0304-3800(94)90017-5

[32] Lande, R. (1988) Genetics and Demography in Biological Conservation. Science, 241, 1455-1460. https://doi.org/10.1126/science.3420403

[33] Lamont, B.B., Klinkhamer, P.G.L. and Witkowski, E.T.F. (1993) Population Fragmentation May Reduce Fertility to Zero in Banksia goodii: A Demonstration of the Allee Effect. Oecologia, 94, 446-450. https://doi.org/10.1007/BF00317122

[34] Boukal, D.S. and Berec, L. (2002) Single-Species Models of the Allee Effect: Extinction Boundaries, Sex Ratios and Mate Encounters. Journal of Theoretical Biology, 218, 375-394. https://doi.org/10.1006/jtbi.2002.3084

[35] Holling, C.S. (1959) The Components of Predation as Revealed by a Study of Small Mammal Predation of the European Pine Sawfly. The Canadian Entomologist, 91, 293-320. https://doi.org/10.4039/Ent91293-5

[36] Holling, C.S. (1959) Some Characteristics of Simple Types of Predation and Parasitism. The Canadian Entomologist, 91, 385-398. https://doi.org/10.4039/Ent91385-7 